INPLASY

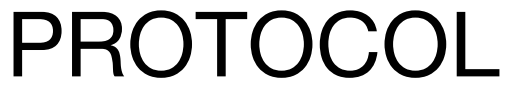

To cite: Sun et al. The acupuncture-related therapy for stable chronic obstructive pulmonary disease: a protocol for systematic review and network meta-analysis. Inplasy protocol 2020120017. doi: 10.37766/inplasy2020.12.0017

Received: 02 December 2020

Published: 03 December 2020

Corresponding author: Haiju Sun

sunhaiju0411@qq.com

Author Affiliation: The Third Clinical Medical College, Zhejiang Chinese Medical University

Support: NKRDPOC (NO. 2018YFC1704600).

Review Stage at time of this submission: Preliminary searches.

Conflicts of interest:

None.

\section{The acupuncture-related therapy for stable chronic obstructive pulmonary disease: a protocol for systematic review and network meta-analysis}

Sun, $\mathrm{H}^{1}$; Zhang, Y2; Lou, J3; Li, X4; Fang, J5.

Review question / Objective: To compare the effectiveness of different acupuncture-related therapies (ATs) for stable chronic obstructive pulmonary disease(SCOPD).

Condition being studied: SCOPD is associated with high incidence, mortality, and disability rates, and presents a heavy economic burden. In recent years, randomized clinical trials of acupuncture-related therapies were gradually grown, and the treatment regimen of SCOPD has been increasingly diversified. However, the quality of acupuncture-related researches is uneven, and the guideline for acupuncturerelated therapies of SCOPD has not yet been developed. The evidence strength of relative studies is somewhat inadequate in guiding the treatment of SCOPD.

INPLASY registration number: This protocol was registered with the International Platform of Registered Systematic Review and Meta-Analysis Protocols (INPLASY) on 03 December 2020 and was last updated on 03 December 2020 (registration number INPLASY2020120017).

\section{INTRODUCTION}

Review question / Objective: To compare the effectiveness of different acupuncturerelated therapies (ATs) for stable chronic obstructive pulmonary disease(SCOPD).
Condition being studied: SCOPD is associated with high incidence, mortality, and disability rates, and presents a heavy economic burden. In recent years, randomized clinical trials of acupuncturerelated therapies were gradually grown, and the treatment regimen of SCOPD has 
been increasingly diversified. However, the quality of acupuncture-related researches is uneven, and the guideline for acupuncture-related therapies of SCOPD has not yet been developed. The evidence strength of relative studies is somewhat inadequate in guiding the treatment of SCOPD.

\section{METHODS}

Search strategy: We will search the following sources without restrictions for date, language, or publication status: PubMed, Cochrane Library, EMBASE, China Biomedical Literature Database(CBM), Chinese National Knowledge Infrastructure (CNKI), and Wanfang Data. We will apply a combination of Medical Subject Heading (MeSH) and free-text terms incorporating databasespecific controlled vocabularies and text words to implement search strategies. Besides, the previous relevant reviews conducted on acupuncture-related therapies for SCOPD and reference lists of included studies will also be searched.

Participant or population: Eligibility of participants will be defined as patients with stable COPD diagnosed by acknowledged criteria. We will apply no restrictions in terms of gender, age, region, or other demographic characteristics.

Intervention: Acupuncture-related therapies are defined as any acupuncture modalities that commonly used in clinical practice, including manual acupuncture, electroacupuncture, acupoint injection, acupoint application, acupoint catgut embedding, warm needling, moxibustion, and other types, regardless of acupuncture materials, acupuncture techniques and stimulation methods.

Comparator: Control interventions will include standard treatments for SCOPD (eg. bronchodilator, expectorant, oxygen therapy, etc), no treatment or waiting-list, and sham controls (eg. sham acupuncture).

Study designs to be included: This review will be confined to all randomized controlled trials (RCTs) involving acupuncture-related therapies for treating stable COPD.

Eligibility criteria: Any diagnostic criteria for SCOPD.

Information sources: Six major Chinese and English databases will be searched, including PubMed, Cochrane Library, Embase, China Biomedical Literature Database, Chinese National Knowledge Infrastructure, and Wanfang Database. Searching dates: from the establishment date to November 2020. A combination of Medical Subject Heading (MeSH) and freetext terms incorporating database-specific controlled vocabularies and text words will be applied to search eligible studies in these electronic databases. In addition, the previous relevant reviews regarding ATs for SCOPD and reference lists of included studies will also be searched to identify additional studies.

Main outcome(s): (1) Pulmonary Function: forced expiratory volume in one second/ prediction (FEV1\% pre) and forced expiratory volume in one second/forced vital capacity (FEV1/FVC\%). (2) Incidence of acute exacerbations (AEs).

Additional outcome(s): (1) The six-minute walking distance (6MWD). (2) St George's Respiratory Questionnaire (SGRQ). (3) The COPD Assessment Test (CAT).

Data management: Two independent reviewers will extract the data from eligible studies using a standardized data extraction form. Data items from each included study will be extracted as follows: study characteristics: study title publication year, first author, journal, country, method of randomization method, blinding method, participant characteristics, interventions of the treatment and control group, primary and secondary outcome measures, etc. If there is any inconsistency between 2 reviewers during the data extraction, it will be verified by a third reviewer. 
Quality assessment / Risk of bias analysis: The methodological quality for each included study will be evaluated using the Cochrane risk-of-bias-tool by two reviewers independently. Any disagreements will be resolved by a judgment of the third reviewer.

Strategy of data synthesis: Before data synthesized, the level of heterogeneity between included studies will be measured by Cochrane $Q$ statistic and the value of $\mathbf{I}^{2}$. If $I^{2} \leq 50 \%$ and $P \geq 0.05$, it will suggest that heterogeneity is not important, in which case the fixed-effect model will be selected for meta-analysis. If $I^{2}>50 \%$ and $P<0.05$, it will mean that the heterogeneity is significant, where the random-effect model will be selected for meta-analysis. Mean difference with $95 \%$ confidence intervals (CI) will be calculated for continuous variable data (e.g. FEV1\%, FEV1/FVC\%, AE, 6MWD, SGRQ, and CAT). Odds ratio (OR) or relative risk (RR) and associated $95 \% \mathrm{Cl}$ will be analyzed for categorical variable data. NMA will be performed using the network command in Stata software (Stata V.14.0, StataCorp) to rank probabilities of different interventions. A network diagram will be made to visualize the numbers and interrelations of different AT interventions. The AT interventions results will be ranked according to their corresponding surface under the cumulative ranking curve, by evaluating the certain extent of intervention superiority without resampling. The evaluation of the inconsistency between the direct and indirect comparison results will be based on the Z-test, the results of which will be displayed via a network graph.

Subgroup analysis: In order to evaluate the possible sources of heterogeneity, subgroup analysis will be conducted. Subgroup analyses will analyze the possible factors that might contribute to heterogeneity, such as intervention in the experimental group (different acupoints), control group (pharmacologic agents, placebo, and sham acupuncture), the duration of treatment, or the standards of the reports under consideration.
Sensibility analysis: Sensitivity analysis will be implemented by excluding studies of the high risk of bias. Lastly, publication bias will be determined based on the funnel plots and Egger regression tests if sufficient numbers of RCTs are included.

Language: Language restrictions will not be imposed.

Country(ies) involved: China.

Keywords: acupuncture, network metaanalysis, stable chronic obstructive pulmonary disease, systematic review

Contributions of each author:

Author 1 - Haiju Sun.

Author 2 - Yajun Zhang.

Author 3 - Jiali Lou.

Author 4 - Xiaoyu Li.

Author 5 - ianqiao Fang. 